Revista Ingeniería y Región. 2015;14(2):125-142

\title{
Ajustes de distribuciones probabilísticas para la variable temperatura media multianual para el departamento de Boyacá (Colombia)
}

\section{Adjust of the probabilistic distribution for the multi-year average temperature variable for the department of Boyacá (Colombia)}

Dayam Soret Calderón Rivera ${ }^{1}$, Claudia Fernanda Navarrete López² y José Luis Díaz Arévalo ${ }^{3}$

\begin{abstract}
Resumen
En este trabajo se presenta un estudio acerca de la selección de la mejor distribución probabilística para la variable media multianual de la temperatura en el departamento de Boyacá (Colombia), como base para futuras estimaciones y proyecciones de la variable en condiciones de incertidumbre. Se seleccionaron las distribuciones Normal, Gamma, Weibull y LogNormal para ajustar los datos; y para encontrar cual distribución ajusta mejor los datos se utilizaron los criterios de información basados en la máxima verosimilitud de Akaike (Akaike Information Criterion) y Bayesiano (Bayesian Information Criteron). Se muestran los resultados tanto en forma tabular como gráfica, así como un plano de las funciones de distribución probabilísticas más representativas en el área de estudio. Como resultado se obtiene que en general la distribución que mejor se ajusta es la Weibull.
\end{abstract}

Palabras clave: Distribuciones de probabilidad; Temperatura; Criterios de información; Boyacá.

\begin{abstract}
This paper presents a study on the selection of the best probabilistic distribution for multi-year average temperature variable in the department of Boyacá (Colombia) as the basis for future estimates and projections of the variable under uncertainty arises. Normal, Gamma, Weibull and lognormal distributions were selected to fit the data. To find which best fits the data criteria information based in maximum likelihood, Akaike (Akaike Information Criterion) and Bayesian (Bayesian Information Criteron) were used. The results are
\end{abstract}

1 Estudiante Facultad de Ingeniería Ambiental. Universidad Santo Tomás (Colombia). Carrera 9 51-11, Bogotá (colombia). E-mail: dayamcalderon@usantotomas.edu.co

2 Magister en Agronomía. Universidad Santo Tomás (Colombia). Carrera 9 51-11, Bogotá (colombia). E-mail: claudianavarrete@usantotomas.edu.co

3 Doctor Ingeniería Hidráulica y Medio Ambiente. Universidad Santo Tomás (Colombia). Carrera 9 51-11, Bogotá (colombia). E-mail: jluisdiaz@usantotomas.edu.co 
shown in both tabular and graphical form, as well as a map of the probabilistic distribution functions most representative in the study area. As a result we obtain that the Weibull distribution is the best fits in general.

Key words: Probabilistic Distributions; Temperature; Information criterion; Boyacá.

\section{Introducción}

El objeto de éste trabajo es determinar la distribución probabilística de mejor ajuste para los datos medios multianuales de la variable climatológica temperatura, en el departamento de Boyacá (Colombia). Estos datos fueron tomados del catálogo de estaciones climatológicas proporcionado por el IDEAM. Se probaron las distribuciones Normal, Gamma, Weibull y LogNormal para ajustar los datos (Navarrete, 2008; Minka 2002; Aristizabal, 2012; Martínez, 2011); y para encontrar cual distribución ajusta mejor los datos se utilizaron los criterios de información de Akaike (AIC) (Akaike, 1974) y Bayesiano (BIC) (Akaike, 1978; Schwarz 1978). Un aspecto importante de los criterios de información es que estos están asociados con el método de máxima verosimilitud, el cual, en modelos causales, está basado en el supuesto de que las variables observadas siguen una distribución normal multivariante (Caballero, 2011). La idea clave del AIC es la de penalizar un exceso de parámetros ajustados, mientras que en el BIC se penaliza el número de parámetros teniendo en cuenta que el AIC podría no ser asintóticamente justificable (Caballero, 2011; Montesinos, 2011; Ramírez, 2000).

Se encontró escasa información aplicada a series de datos hidrológicos (Ayodele, 2015; Chaouche, et.al., 2010; Donat, 2012; Räisänen, 2009; Hingray, 2007), entre los cuales a nivel local se destaca el trabajo presentado por Dorado et. al. en el 2006 donde analizaron la variabilidad espacio-temporal de la precipitación mensual y anual en el área de influencia aguas abajo del embalse de regulación y abastecimiento SARABRUT, ubicado en el departamento del Valle del Cauca, Colombia. Para el ajuste de las series históricas se usaron modelos de distribución de probabilidad teórica, como Gumbel, Normal, LogNormal y Wakeby, y modelos de distribución empírica, como Weibull y Landwehr. Se utilizó la prueba de Error Estándar de Ajuste (EEA) para seleccionar el modelo teórico de mejor ajuste. El modelo Wakeby presentó el mejor ajuste en el $89 \%$ del total de estaciones analizadas, el cual corresponde a un modelo probabilístico adecuado para representar los caudales de crecidas máximas diarias o instantáneas en una región. No obstante para nuestro estudio, la distribución Weibull se presenta como la más confiable debido a su versatilidad (Behary Grimas, 2004), (Moreno et al., 2011). En su trabajo de maestría (Navarrete, 2008) encuentra que para los criterios de AIC y BIC la distribución Weibull es la que mejor describe los datos.

\section{Metodología}

Este trabajo fue realizado sobre la totalidad de la extensión del departamento de Boyacá a partir de la información proporcionada por el Instituto de Hidrología, Meteorología y Estudios Ambientales de Colombia (IDEAM), el cual cuenta con 35 estaciones meteorológicas en la región, las cuales se presentan en la tabla 1 junto con la extensión de la serie analizada. De estas estaciones se obtuvo el promedio multianual de la variable temperatura, y se realizaron los histogramas de frecuencia para cada una de las estaciones con la finalidad de determinar gráficamente las funciones a ajustar. No se consideró necesario realizar completado de datos ya que la variable temperatura media presenta poca varianza $(0.52$ en promedio) y el porcentaje de datos faltantes global es bajo (3.60\%).

Se aplicaron las pruebas de Mann-Kendall (Mann, 1945; Kendall, 1975) y Rachas con la finalidad de validar supuestos de homogeneidad y aleatoriedad de las estaciones muestreadas. Los resultados obtenidos mostraron que la mitad de las estaciones presentan un comportamiento homogéneo y aleatorio. Como el objetivo del trabajo es presentar las funciones de distribución de mejor ajuste a las series de datos, no se realizaron transformaciones a las mismas.

Por medio de los software $\mathrm{R}^{4}$ y ParameterSolver $^{5}$ se procesaron los datos y se estimaron los parámetros, con el fin de hallar las distribuciones de probabilidady las funciones de máxima verosimilitud, las cuales fueron utilizadas para calcular los criterios de información Akaike (AIC) y Bayesiano (BIC); con base en estos se escogió la distribución de mejor ajuste. El AIC fue propuesto por Akaike, H. (1974) como un estimador insesgado asintótico de la información de KullbackLeibler esperada entre un modelo candidato ajustado y el verdadero modelo. El BIC fue derivado por Akaike (1978) y Schwarz (1978) como una aproximación a 
Tabla 1. Estaciones climatológicas

\begin{tabular}{c|c|c|c|c|c}
\hline Nombre & Código & Años & Nombre & Código & Años \\
\hline PTO BOYACA & 23115010 & 39 & TUNEL EL & 35095030 & 42 \\
\hline OTANCHE & 23125080 & 39 & UPTC & 24035130 & 45 \\
\hline Buena Vista & 23125100 & 20 & SURBATA BONZA & 24035120 & 60 \\
\hline APTO FURATENA & 23125140 & 23 & TUNGUAVITA & 24035170 & 46 \\
\hline INST AGR SANTA SOFIA & 24015090 & 28 & CHITA & 24035250 & 42 \\
\hline VILLA CARMEN & 24015220 & 46 & SATIVANORTE & 24035320 & 39 \\
\hline VILLA DE LEIVA & 24015300 & 34 & NUEVO COLON & 35075010 & 48 \\
\hline SIERRA LA & 24025030 & 47 & INST AGR MACANAL & 35075040 & 32 \\
\hline CUSAGUI & 24035010 & 28 & CAMPOHERMOSO & 35085050 & 27 \\
\hline BETEITIVA & 24035010 & 23 & CARDON & 35235010 & 39 \\
\hline COPA LA & 24035040 & 22 & BELENCITO & 24035150 & 47 \\
\hline GUICAN & 24035070 & 23 & COCUY & 24035240 & 40 \\
\hline APTO A LLERAS C & 24035340 & 32 & ANDALUCIA & 24035350 & 18 \\
\hline CHISCAS & 24035310 & 40 & VILLA LUISA & 35075030 & 29 \\
\hline RONDON & 35085020 & 42 & VIVERO & 35085040 & 30 \\
\hline SAN RAFAEL & 24035180 & 34 & CORINTO & 35195050 & 29 \\
\hline SUTATENZA & 35075020 & 44 & TUNEBIA & 37035010 & 40 \\
\hline BOAVITA & 24035330 & 36 & & & \\
\hline
\end{tabular}

Fuente: Catálogo red estaciones hidroclimaticas - IDEAM.

una transformación de la probabilidad posterior de un modelo candidato.

Las distribuciones de probabilidad y los criterios se describen a continuación:

\section{Distribuciones de probabilidad}

Distribución normal: esta distribución de probabilidad continua es la más importante y la más utilizada, también llamada curva de Gauss. Esta dada por dos parámetros de la función $\mathrm{f}(\mathrm{i}, \mathrm{o})$, donde ó representa la desviación estándar y $\mu$ la media de la muestra

$$
f(x)=\frac{1}{\sigma \sqrt{2 \pi}} \mathrm{e}^{-\frac{(x-\mu)^{2}}{2 \sigma^{2}}}
$$

Distribución Gamma: es un caso especial de la distribución Pearson tipo III donde sus parámetros deben ser diferentes de 0. Esta distribución está dada por: Ã(á) la función gamma de á, â como el parámetro de escala y á es el parámetro de forma.

$$
\begin{gathered}
f(x)=\frac{1}{\Gamma(\alpha) \beta^{\alpha}} \mathrm{x}^{(\alpha-1)} \mathrm{e}^{-\frac{\mathrm{x}}{\beta}} \\
\text { donde } \alpha=\frac{1}{\left(\frac{\sigma}{\mu}\right)^{2}} \text { y } \beta=\frac{\mu}{\sigma^{2}}
\end{gathered}
$$

Distribución Weibull: esta distribución es muy versátil y es utilizada para modelar amplia gama de aplicaciones, describiéndose según los parámetros de escala y localización â, forma m y á como origen de la distribución.

$$
f(x)=\frac{m}{\beta}\left(\frac{x-\alpha}{\beta}\right)^{m-1} \mathrm{e}^{-\left(\frac{x-\alpha}{\beta}\right)^{m}}
$$

$$
\text { donde } \alpha=\frac{1}{n} \sum_{\mathrm{i}=1}^{\mathrm{n}}\left(\mathrm{xi}^{\beta}\right)^{1 / \beta} \text { y } \beta=\frac{\mu}{\sigma}
$$

Distribución Log normal: al igual que la distribución normal está dada por los dos parámetros donde ó representa la desviación estándar y $\mu$ la media de la muestra

$$
f(x)=\frac{1}{\mathrm{x} \sigma \sqrt{2 \pi}} \mathrm{e}^{-\left(\frac{(\ln (\mathrm{x})-\mu)^{2}}{2 \sigma^{2}}\right)}
$$

\section{Máxima verosimilitud}

La función de máxima de verosimilitud está dada por la máxima probabilidad de que ocurra o se dé una determinada muestra si es cierta la estimación 
que hemos efectuado, o el estimador que hemos planteado. La densidad conjunta para esas observaciones es denotada como $L=f(x i, p)$, y la máxima verosimilitud puede ser obtenida tomando la derivada de $L$ en relación con la probabilidad $p$ igualando la función a 0 , para lo cual es mejor tomar logaritmos de la función $L$ y derivarlo; $n$ es el número de observaciones.

Normal

$$
\ln (x) L\left(x, \mu, \sigma^{2}\right)=-n \ln (x)(\sigma \sqrt{2 \pi}) \frac{\sum_{\mathrm{i}=1}^{\mathrm{n}}(\mathrm{xi}-\mu)}{2 \sigma^{2}}
$$

Gamma

$$
\ln (x) L(x, \alpha, \beta)=n \alpha \ln (\beta)-n \ln [\Gamma(\alpha)]+(\alpha-1) \sum_{\mathrm{i}=1}^{\mathrm{n}}(\ln (\mathrm{xi}))-\beta \sum_{\mathrm{i}=1}^{\mathrm{n}}(\mathrm{xi})
$$

Weibull

$$
\ln (x) L(x, \alpha, \beta)=n[\ln (\alpha)-\alpha \ln (\beta)]+(\alpha-1) \sum_{\mathrm{i}=1}^{\mathrm{n}}(\ln (\mathrm{xi}))-\frac{1}{\beta^{\alpha}} \sum_{\mathrm{i}=1}^{\mathrm{n}}\left(\mathrm{xi}^{2}\right)
$$

Log-normal

$$
\ln (x) L\left(x, \mu, \sigma^{2}\right)=-\frac{n}{2} \ln \left(2 \pi \sigma^{2}\right)-\sum_{\mathrm{i}=1}^{\mathrm{n}}(\ln (\mathrm{xi}))-\frac{\sum_{\mathrm{i}=1}^{\mathrm{n}}\left(\ln (\mathrm{xi})^{2}\right)}{2 \sigma^{2}}+\frac{\sum_{\mathrm{i}=1}^{\mathrm{n}}(\mu \ln (\mathrm{xi}))}{\sigma^{2}}-\frac{n \mu^{2}}{2 \sigma^{2}}
$$

\section{Calculo $A I C$ y $B I C$}

El criterio de información Akaike propuesto en 1974 y utilizado como un estimador insesgado, precisa que el modelo de probabilidad de menor valor AIC es el que se selecciona como el mejor al que se le ajustan los datos. La función está dada por la maximización del logaritmo de la máxima verosimilitud denotado como (ln $L$ ) y $K$ es el número de parámetros de la función de probabilidad (parámetros en el modelo):

$$
A I C=2 \mathrm{~K}-2 \ln (\mathrm{L})
$$

La estructura del AIC está compuesta por la maximización del logaritmo de verosimilitud, es decir, como componente de la falta de ajuste del modelo y $K$ como el número de parámetros estimados dentro del modelo como componente de penalidad. La penalidad es una medida de la complejidad o compensación por el sesgo debido a la falta de ajuste cuando los estimadores de máxima verosimilitud son empleados (Bozdogan, 1987 en Acuña et. al., 2012).

BIC sirve para la selección del modelo entre un conjunto finito de modelos, está estrechamente relacionado con el criterio AIC y se basa en parte en la función de probabilidad. Para mejorar la inconsistencia del cri- terio AIC, Akaike 1978 y Schwarz 1978 presentaron un criterio de selección de modelos desde la perspectiva bayesiana. Schwarz estableció que la solución de bayes consiste en seleccionar el modelo con una alta probabilidad a posteriori. El criterio de información bayesiana (BIC) se define como:

$$
B I C=2 \ln (L)+K \ln (n)
$$

Mapa de resultados

Una vez obtenidos los criterios AIC y BIC para cada una de las distribuciones por estación se procedió a realizar el mapa de área de influencia como se muestra en la figura 2.

\section{Resultados}

Después de obtener las distribuciones de probabilidad para cada una de las estaciones y de la aplicación de los criterios AIC y BIC se observó que la distribución dominante en el departamento de Boyacá es la Weibull ajustándose a 21 estaciones de las 35 muestreadas $(60 \%)$, en segundo lugar se encontraron las distribuciones Normal y Gamma con 6 estaciones cada una $(17 \%)$ y por último la distribución Lognormal con 2 estaciones (6\%) (tabla 2$)$. 
Ajustes de distribuciones probabilisticas parala variable temperatura .../ Calderón Rivera et al. Revista Ingeniería y Región. 2015;14(2):9-18

Tabla 2. Criterios de ajuste de distribución

\begin{tabular}{|c|c|c|c|c|c|c|c|c|}
\hline \multirow{2}{*}{$\begin{array}{c}\text { Nombre de la } \\
\text { estación }\end{array}$} & \multicolumn{4}{|c|}{ AIC } & \multicolumn{4}{|c|}{ BIC } \\
\hline & Normal & Gamma & Weibull & $\begin{array}{c}\log \\
\text { normal }\end{array}$ & Normal & Gamma & Weibull & $\begin{array}{c}\text { Log } \\
\text { normal }\end{array}$ \\
\hline PTO BOYACA & 234,7532 & 237,8511 & 205,2972 & 239,3974 & 231,4261 & 234,524 & 201,9701 & 236,0703 \\
\hline OTANCHE & 340,4676 & 351,6669 & 277,1768 & 356,6061 & 337,1405 & 348,339 & 273,8497 & 353,2790 \\
\hline BUENA VISTA & 58,12972 & 58,25276 & 59,98834 & 58,94445 & 56,13826 & 56,26130 & 57,99688 & 56.95299 \\
\hline $\begin{array}{c}\text { APTO } \\
\text { FURATENA }\end{array}$ & 63,93424 & 63,03625 & 77,63782 & 63,01264 & 61,66325 & 60,76526 & 75,36683 & 60,74165 \\
\hline $\begin{array}{l}\text { INST SANTA } \\
\text { SOFIA }\end{array}$ & 78,27692 & 78,43149 & 86,12450 & 79,21007 & 75,61252 & 75,76708 & 83,46009 & 76,54567 \\
\hline $\begin{array}{c}\text { VILLA } \\
\text { CARMEN }\end{array}$ & 163,8532 & 165,5435 & 162,7736 & 166,4026 & 167,5105 & 169,2008 & 166,4309 & 170,0599 \\
\hline $\begin{array}{l}\text { VILLA DE } \\
\text { LEIVA }\end{array}$ & $161,665]$ & 164,0032 & 149,5635 & 63,8859 & 158,6124 & 160,9505 & 146,5107 & 160,8332 \\
\hline SIERRA LA & 98,33291 & 97,61236 & 120,10444 & 98,06344 & 94,63261 & 93,91206 & 116,40415 & 94,36314 \\
\hline CUSAGUI & 125,6314 & 127,9963 & 114,8585 & 127,8248 & 122,9670 & 125,3319 & 112,1941 & 125,1604 \\
\hline BETEITIVA & 92,21152 & 93,08549 & 188,54432 & 92,99109 & 89,94054 & 90,81450 & 186,27333 & 90,72010 \\
\hline COPALA & 67,33759 & 66,47118 & 77,71301 & 66,28266 & 65,15551 & 64,28910 & 75,53093 & 64,10058 \\
\hline GUICAN & 42,03726 & 41,40550 & 54,73516 & 41,52515 & 39,76627 & 39,13451 & 52,46418 & 39,25417 \\
\hline $\begin{array}{c}\text { APTO A } \\
\text { LLERAS C }\end{array}$ & 193,5991 & 198,8904 & 168,3923 & 203,7743 & 190,6677 & 195,9589 & 165,4609 & 200,8428 \\
\hline CHISCAS & 272,5039 & 280,2488 & 231,4761 & 278,6346 & 269,1262 & 276,8711 & 228,0984 & 275,2568 \\
\hline RONDON & 125,7769 & 125,6248 & 141,0327 & 126,3589 & 122,3015 & 122,1495 & 137,5574 & 122,8835 \\
\hline SAN RAFAEL & 137.8840 & 139,1904 & 138.7613 & 139,5146 & 134,8313 & 136,1377 & 135.7086 & 136,4618 \\
\hline SUTATENZA & 194,1656 & 195,8829 & 195,0512 & 198,1723 & 190,5973 & 192,3146 & 191,4828 & 194,6039 \\
\hline TUNEL EL & 132,3041 & 132,9302 & 149,5230 & 135,3521 & 128,8287 & 129,4549 & 146,0476 & 131,8768 \\
\hline UPTC & 198,2894 & 201,6581 & 181,5968 & 203,8836 & 194,6761 & 198,0448 & 177,9835 & 200,2703 \\
\hline $\begin{array}{l}\text { SURBATA } \\
\text { BONZA }\end{array}$ & 212,0912 & 211,0693 & 235,2744 & 213,5415 & 207,9025 & 206,8807 & 231,0857 & 209,3528 \\
\hline TUNGUAVITA & 213,9447 & 216,3391 & 191,1763 & 221,1755 & 210,2875 & 212,6818 & 187,5190 & 217,5183 \\
\hline CHITA & 249,9439 & 257,7754 & 159,1011 & 264,3868 & 246,4686 & 254,3001 & 155,6258 & 260,9114 \\
\hline SATIVANORTE & 173.5771 & 177.2878 & 139.4639 & 177.5658 & 170,2499 & 173,9607 & 136.1368 & 174,2386 \\
\hline $\begin{array}{l}\text { NUEVO } \\
\text { COLON }\end{array}$ & 181,1275 & 182,5239 & 152,1226 & 185,2750 & 184,8699 & 186,2663 & 155,8650 & 189,0174 \\
\hline $\begin{array}{l}\text { INST AGR } \\
\text { MACANAL }\end{array}$ & 184,3095 & 187,8479 & 105,5642 & 188,5308 & 181,3780 & 184,9164 & 102,6327 & 185,5994 \\
\hline $\begin{array}{c}\text { CAMPOHERM } \\
\text { OSO } \\
\end{array}$ & 30,31309 & 29,96005 & 41,77312 & 30,03026 & 27,72141 & 27,36837 & 39,18145 & 27,43858 \\
\hline CARDON & 151,3815 & 155,5726 & 108,7090 & 161,1811 & 148,0544 & 152,2455 & 105,3819 & 157,8540 \\
\hline BELENCITO & 146,7130 & 144,3126 & 165,2663 & 144,8319 & 143,0127 & 140,6123 & 161,5660 & 141,1316 \\
\hline COCUY & 216,3229 & 227,7881 & 161,7740 & 230,4454 & 212,9452 & 224,4103 & 158,3962 & 227,0676 \\
\hline ANDALUCIA & 48,51900 & 48,53407 & 43,08183 & 48,15724 & 46,73825 & 46,75332 & 41,30108 & 46,37649 \\
\hline VILLA LUISA & 115,41367 & 116,23370 & 98,95385 & 120,41404 & 112,67908 & 113.49911 & 96,21925 & $\begin{array}{c}117,6794 \\
5 \\
\end{array}$ \\
\hline VIVERO & 111,7589 & 111,8932 & 106,7041 & 113,4887 & 108,9565 & 109,0908 & 103,9017 & 110,6863 \\
\hline CORINTO & 221.7676 & 229,7986 & 183,0025 & 224,4988 & 219,0330 & 227,0640 & 180,2679 & 221,7643 \\
\hline TUNEBIA & 131,1866 & 131,5875 & 109,3331 & 132.2281 & 127.8088 & 128,2097 & 105,9553 & 128,8504 \\
\hline BOAVITA & 128,6716 & 129,3392 & 108,2066 & 130,4852 & 125,5045 & 126,1722 & 105,0396 & 127.3182 \\
\hline
\end{tabular}

Fuente: Autores. Fecha 30 de Octubre de 2015 
A continuación se presentan las figuras de las distribuciones de probabilidad para cada una de las estaciones climatológicas del estudio, en color verde se puede apreciar la distribución Weibull.

Figura 1. Distribuciones de probabilidad de la variable temperatura para cada una de la estaciones.

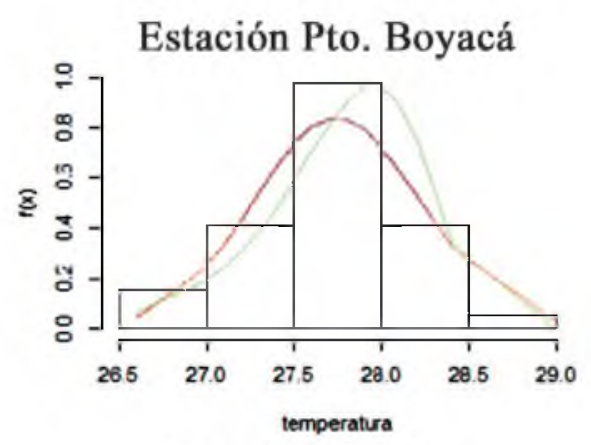

Estación Furatena

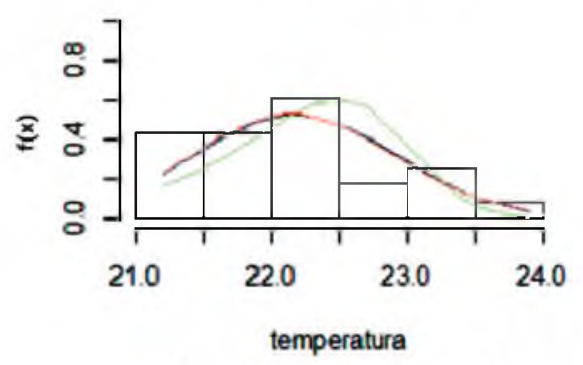

Estación Santa Sofia

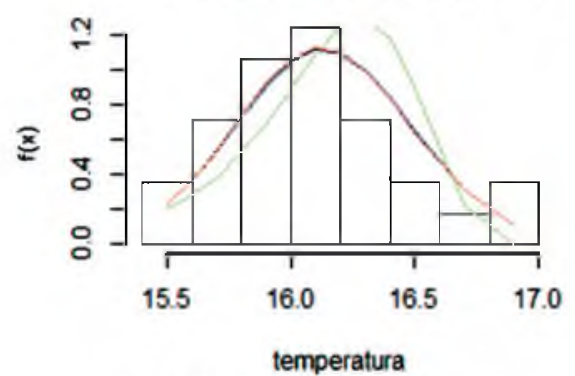

Estación Villa de Leiva

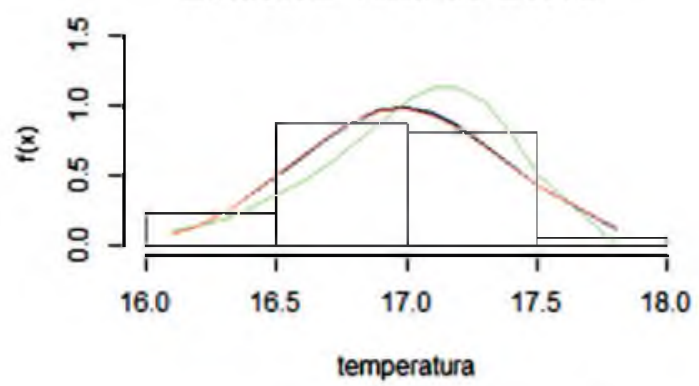

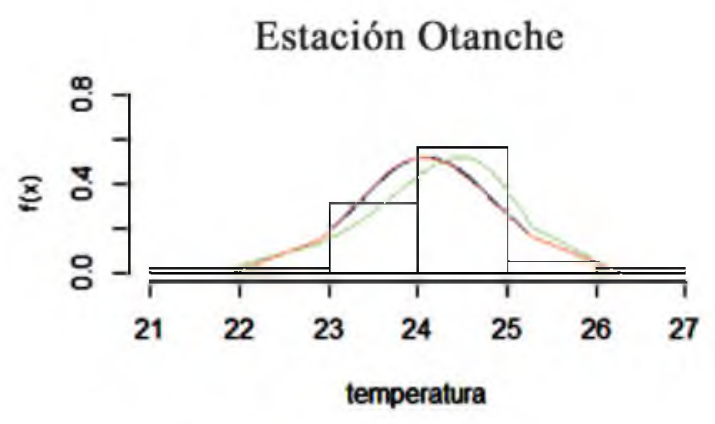

Estación Buena Vista

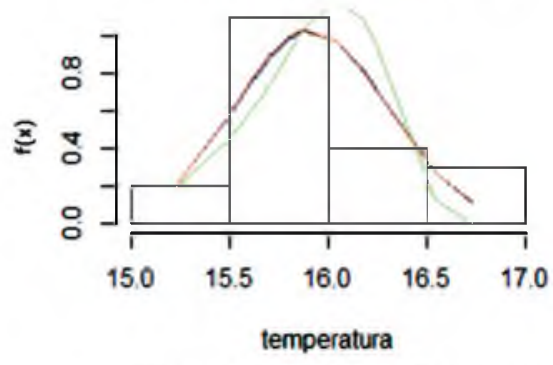

Estación Villa Carmen

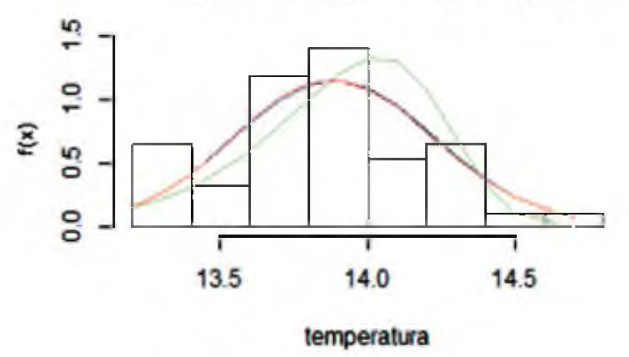

Estación La Sierra

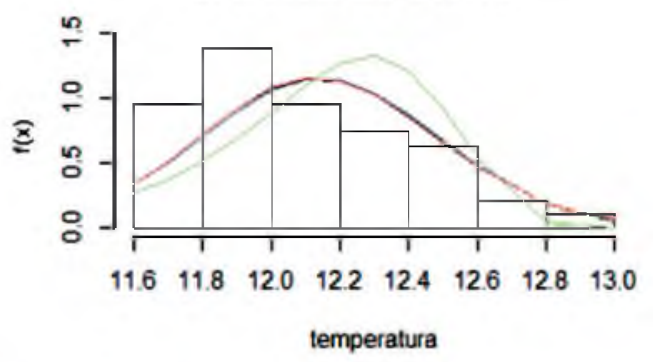

Weibull —Log normal 
Continuación figura 1

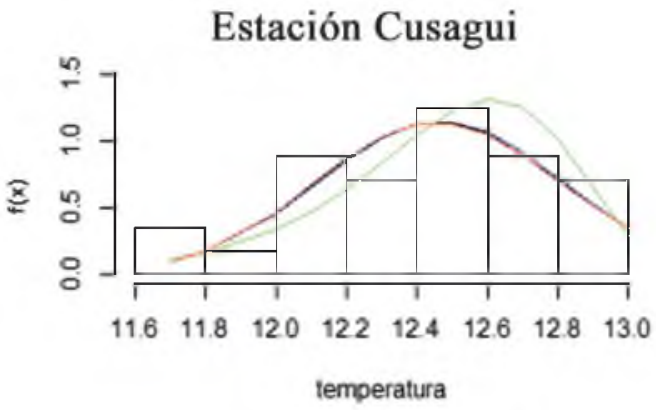

Estación La Copa

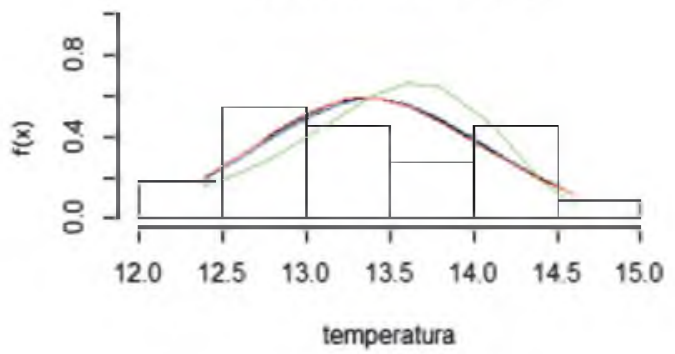

Estación Apto. Lleras

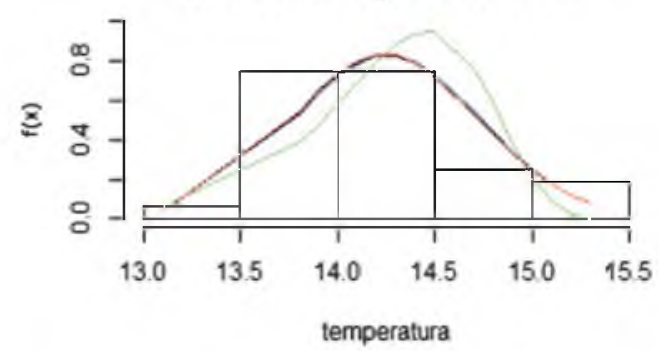

Estación Rondón

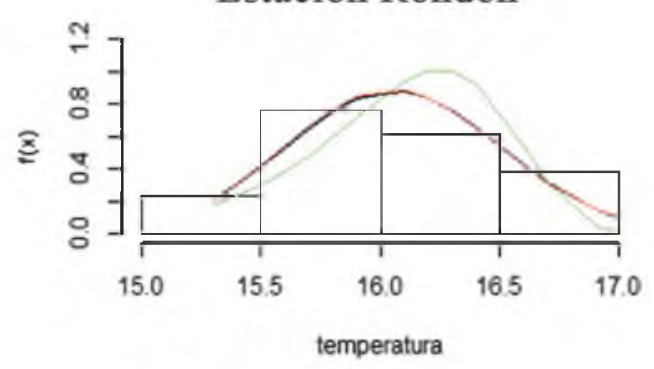

Estación Sutatenza

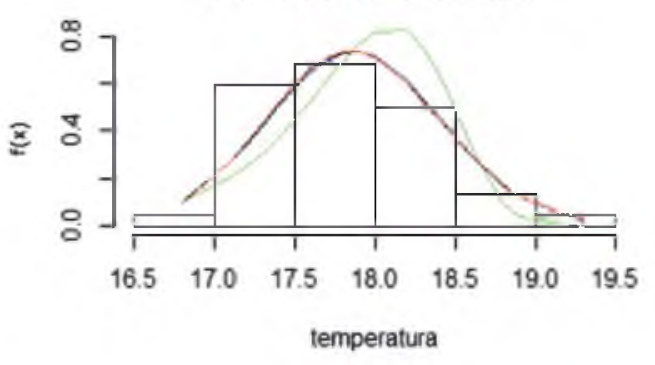

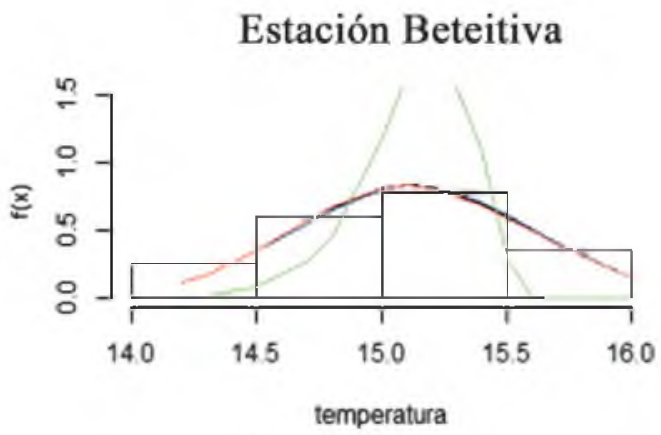

Estación Guicán

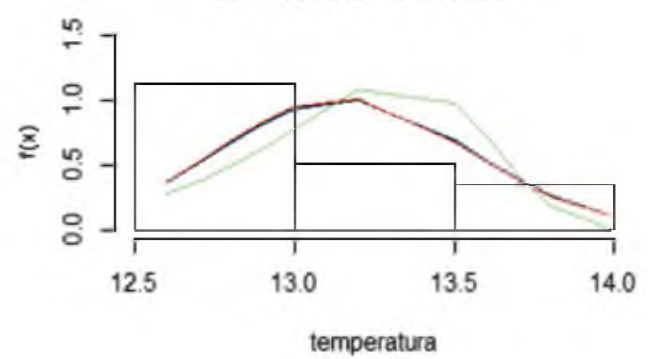

Estación Chiscas
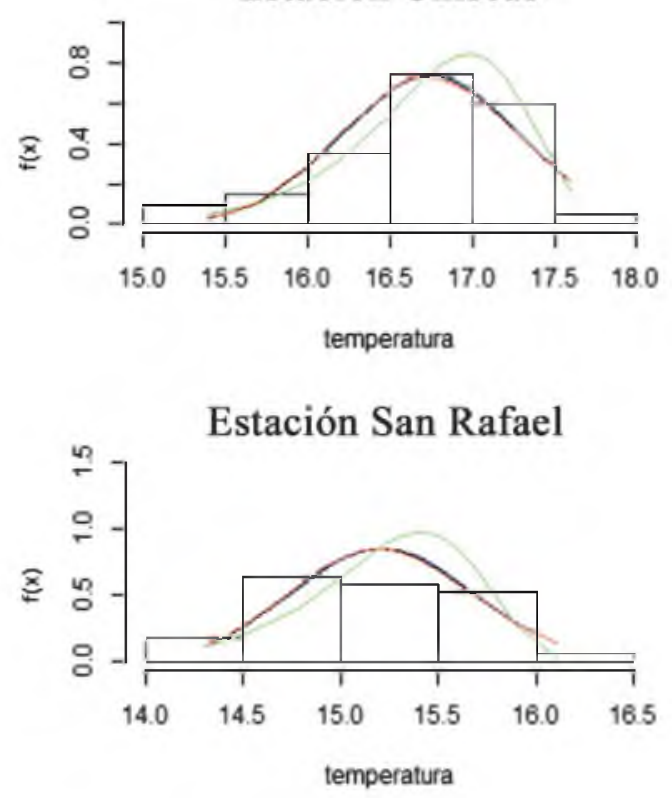

Estación E1 Tunel

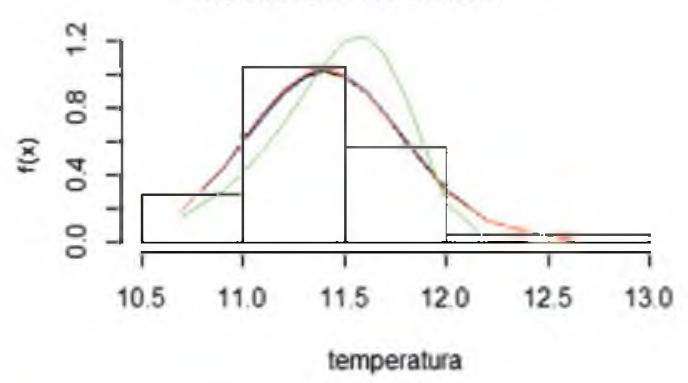

— Norma — Gamma -Weibull —Log normal 


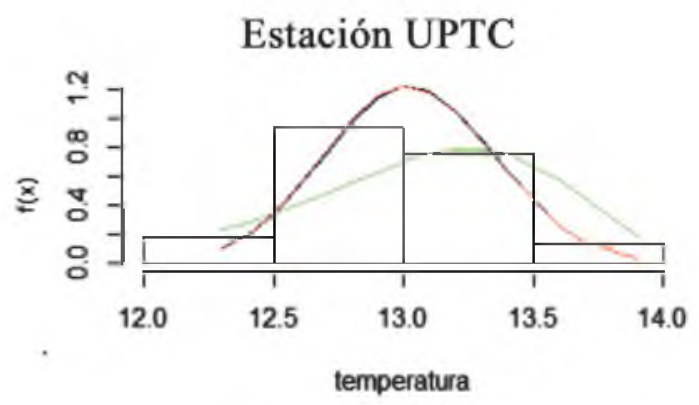

Estación Tunguavita
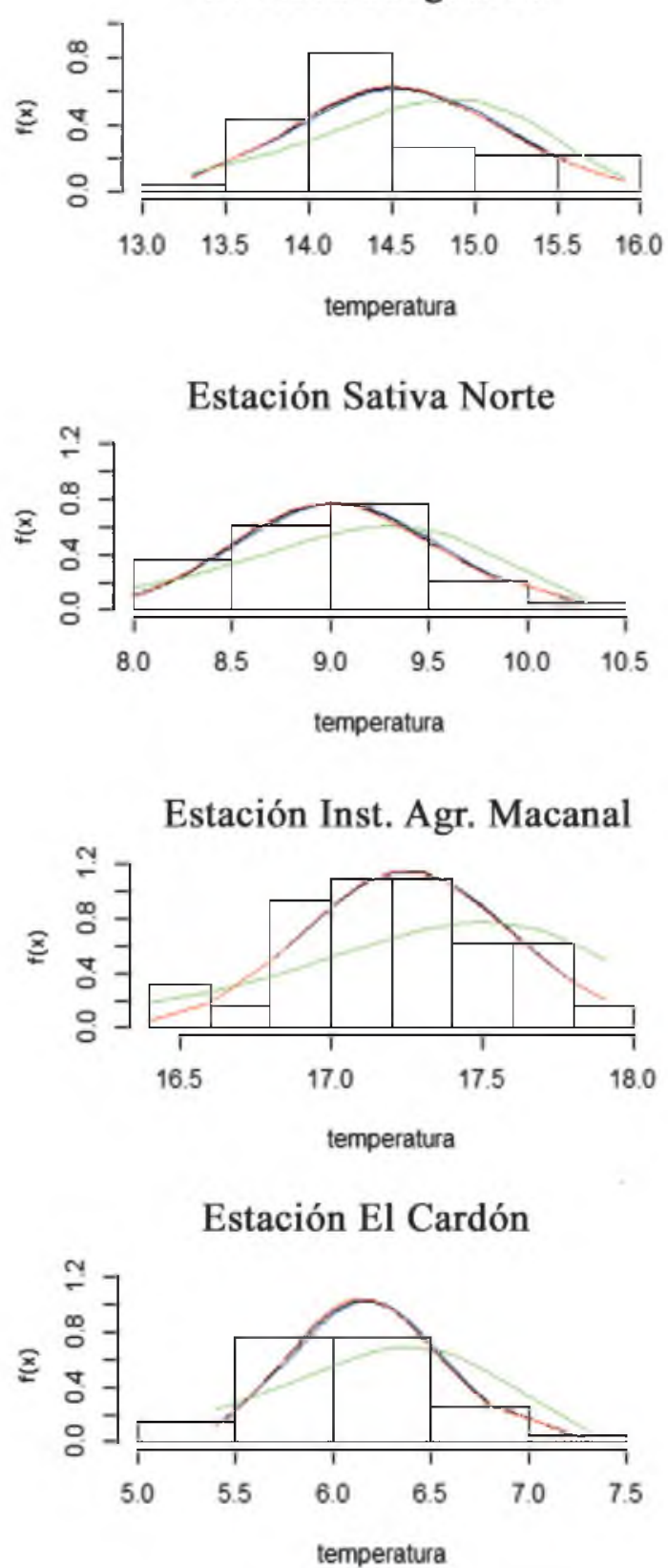
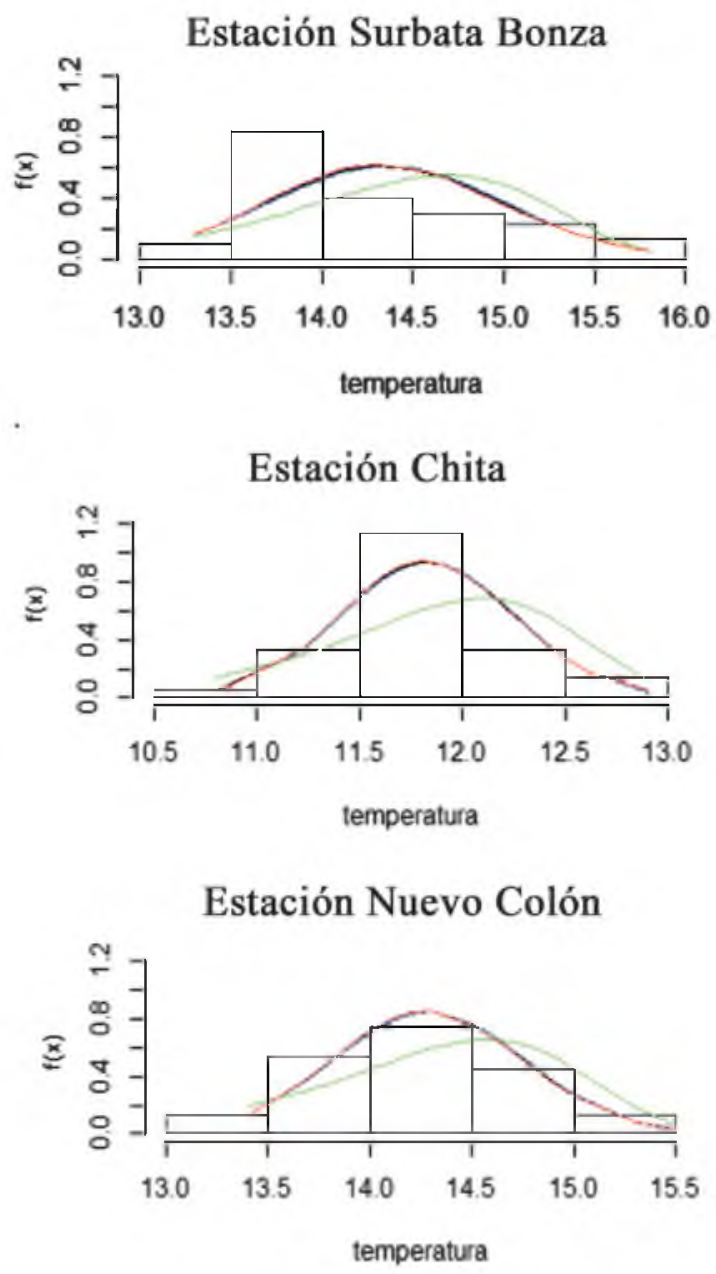

Estación Campo Hermoso
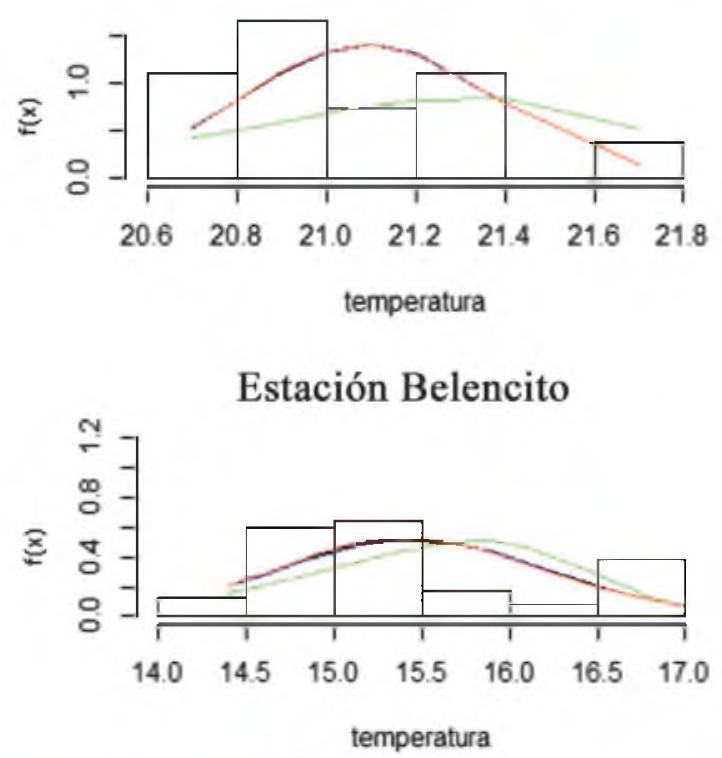

— Norma — Gamma -Weibull —Log normal 
Continuación figura 1

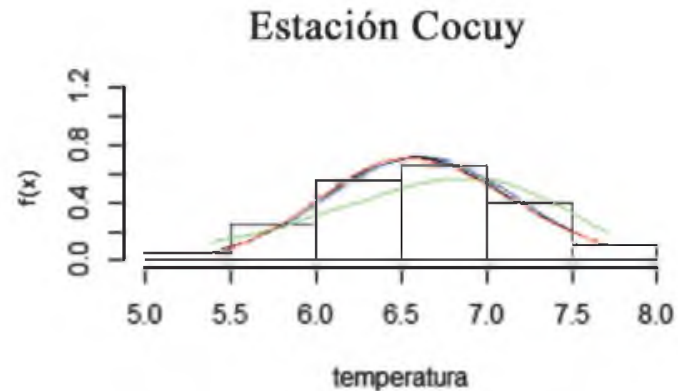

Estación Villa Luisa

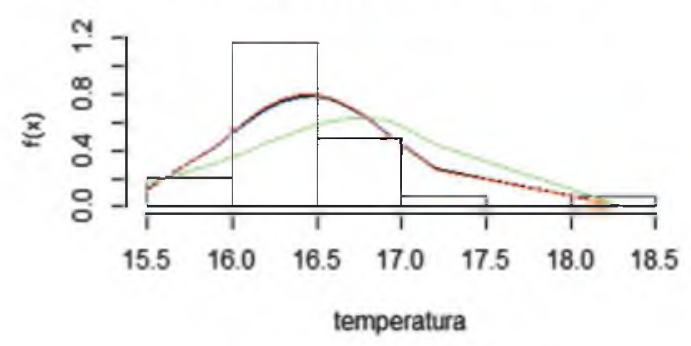

Estación Corinto

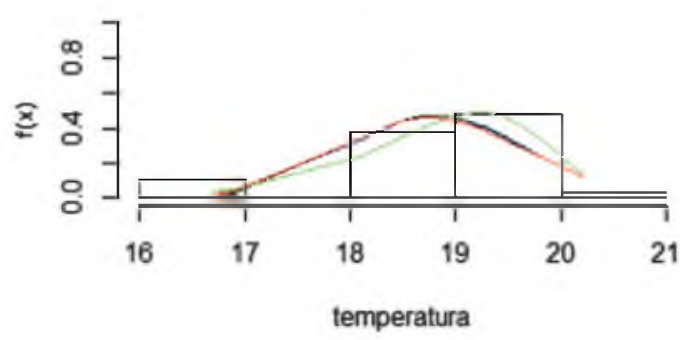

Estación Andalucía

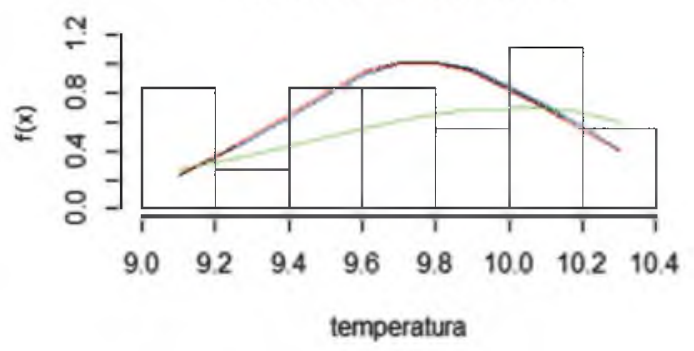

Estación Vivero

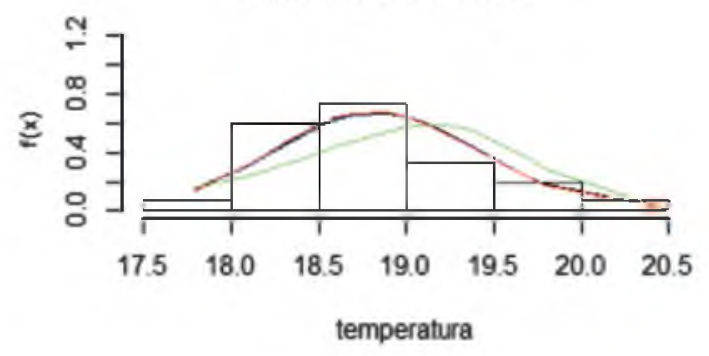

Estación Tunebia

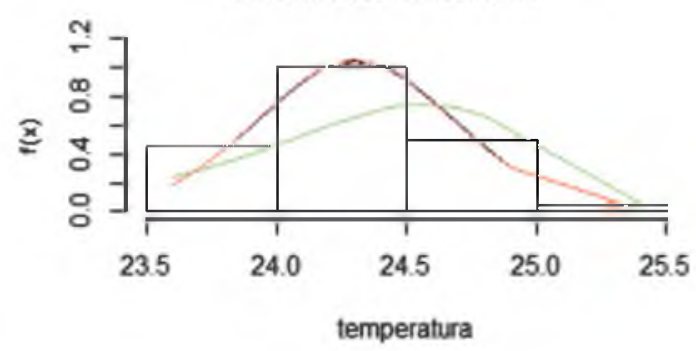

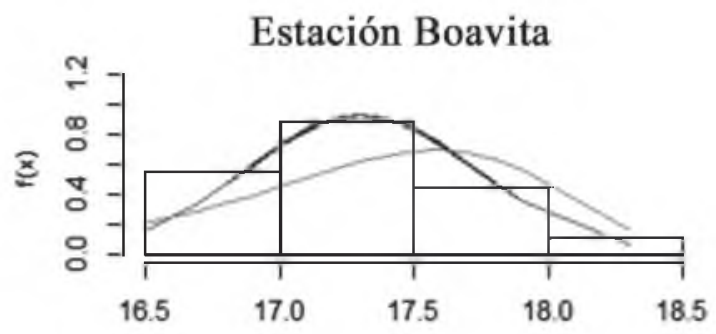

Fuente: Autores. Fecha 30 de noviembre de 2015. 


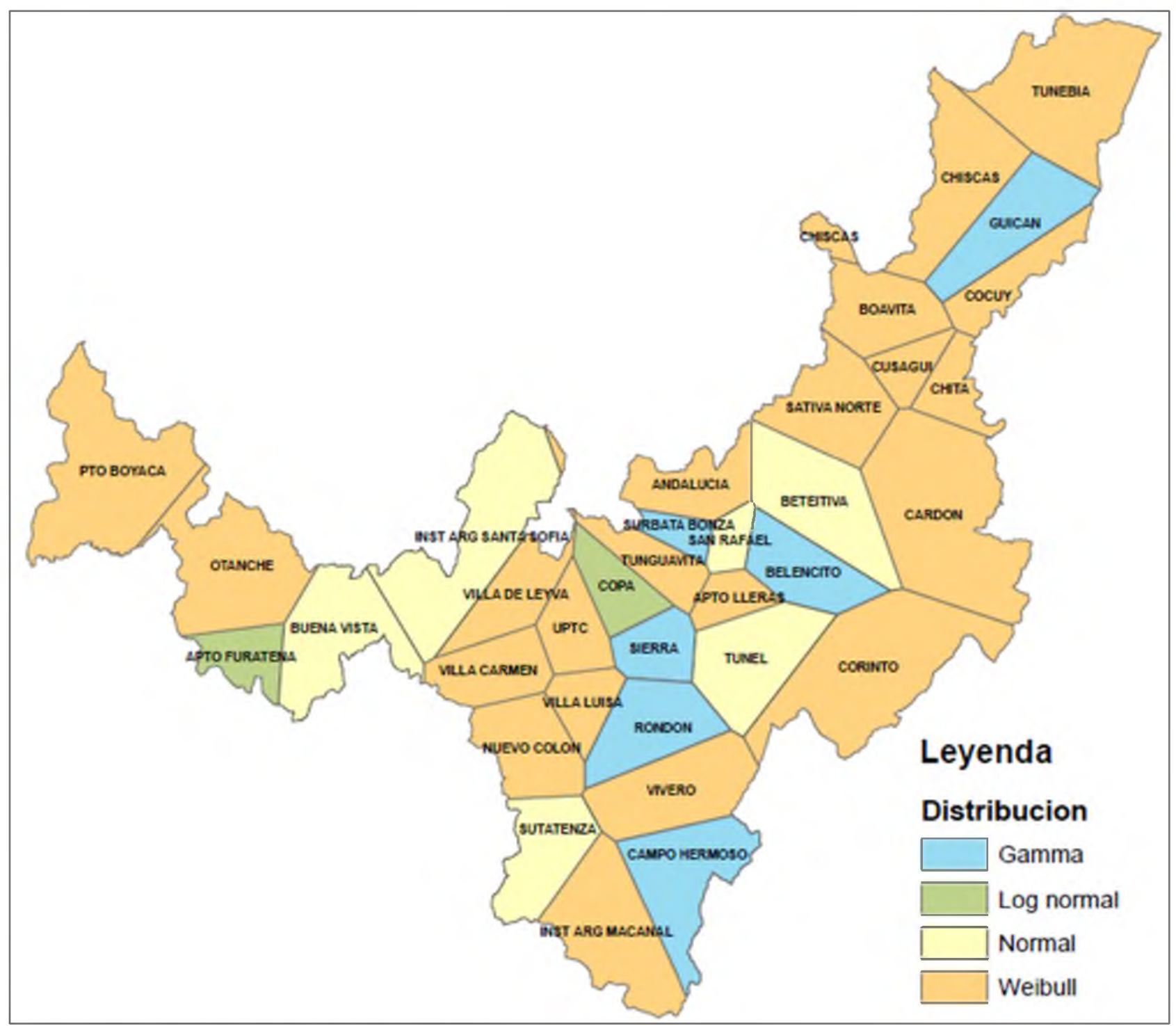

Fuente: Autores. Fecha 30 de noviembre de 2015.

Figura 2. Área de influencia de distribuciones en el departamento de Boyacá.

\section{Conclusiones}

Gráficamente las distribuciones Normal, Gamma y Log normal presentan comportamientos muy similares para la variable temperatura media; la distribución Weibull difiere de estos comportamientos tanto gráficamente así como cuando se aplican los criterios AIC y BIC, tal como se puede apreciar en la figura 1 .

El ajuste según el logaritmo de la función de máxima verosimilitud muestra que distribución Weibull es la más adecuada en la mayoría de las estaciones seguida de las funciones Normal y Gamma.
Los métodos propuestos en el presente trabajo permiten ajustar y determinar de manera sencilla distribuciones probabilísticas continuas aplicadas a la variable de temperatura media, permitiendo futuras estimaciones y proyecciones de la variable en condiciones de incertidumbre.

El $60 \%$ de las estaciones presentan un ajuste a la distribución Weibull, el cual puede estar basado en la versatilidad de dicha función, la cual puede adoptar diferentes formas según sus parámetros.

Las estaciones con menor cantidad de datos tienden ajustarse a distribuciones diferentes a la Weibull, por el contrario cuando las estaciones 
presentan mayor cantidad de datos estas se ajustan mejor a la función de probabilidad Weibull.

- El tamaño de la muestra influye directamente en la confiabilidad de los datos en el cual se trabajo con un mínimo de 18 y un máximo de 60 años para las estaciones analizadas.

\section{Referencias bibliográficas}

Acuña, C., J.A.; Dominguez, C., A.H.; Toro, O., E.M. 2012 Una comparación entre métodos estadísticos clásicos y técnicas metaheurísticas en el modelamiento estadístico. Scientia et Technica Año XVII, No 50, Abril de 2012. Universidad Tecnológica de Pereira. ISSN 0122-1701

Akaike. H. 1974. A new look at the statistical model identification. IEEE Transactions on Automatic Control, 19(6), pp. 716-723.

Akaike. H. 1978. A Bayesian analysis of the minimum AIC procedure. The Annals of Statistics, 30(1), pp. 9-14, 1978.

Aristizabal, R. J. 2012. Estimating the parameters of the three-parameter lognormal distribution. Florida International University. FIU Electronic Theses and Dissertations. Paper 575.

Ayodele, T.R.; Ogunjuyigbe, A.S.O. 2015. Prediction of monthly average global solar radiation based on statistical distribution of clearness index. Energy 90. pp. 1733-1742.

Behar, G., R.; Grima, C., P. 200455 Respuestas a dudas típicas de estadística. Ediciones Díaz de Santos, S. A.

Bozdogan. H. 1987. Model selection and Akaike's information criterion (AIC): The general theory and its analytical extensions. Psychometrika, 52(3), pp. 345-379, 1987.

Caballero, D.; F.F. 2011. Selección de modelos mediante criterios de información en análisis factorial. Aspectos teóricos y computacionales. Tesis Doctoral. Universidad de Granada. Departamento de Estadística e I.O. ISBN 978-84-694-5742-9.

Chaouche, K.; Neppel, L.; Dieulin, C.; Pujol, N.; Ladouche, B.; Martin, E.; Salas, D.; Caballero, Y. 2010. Analyses of precipitation, temperature and evapotranspiration in a French Mediterranean region in the context of climate change. C. R. Geoscience 342. 234-243.

Donat, M.G.; Alexander, L.V. 2012. The shifting probability distribution of global daytime and nighttime temperatures. GEOPHYSICAL RESEARCH LETTERS, VOL. 39, L14707, doi:10.1029/ 2012GL052459, 2012.

Dorado, D., J.; Burbano, C., J.C.; Molina, T., J.M.; Carvajal, E., Y.; Aristizábal, H.F. 2006. Ajuste de modelos probabilísticos para el estudio de la variabilidad espacio-temporal de la precipitación: caso de estudio sistema Sara-Brut. Meteorología Colombiana. No. 10. pp. 60-75. Marzo 2006. ISSN 0124-6984.

Hingray, B.; Mezghani, A.; Buishand, T.A. 2007. Development of probability distributions for regional climate change for uncertain global mean warming and uncertain scaling relationship. Hydrology \& Earth Systems Sciences, 11(3), pp. 1097-1114.

Kendall, M.G. 1975. Rank Correlation Methods, 4th edition, Charles Griffin, London.

Mann, H.B. 1945. Non-parametric tests against trend, Econometrica 13, pp.163-171.

Martínez, F., L. Métodos de inferencia para la distribución Weibull: aplicación en fiabilidad industrial. Trabajo fin de máster. Máster en Técnicas Estadísticas. Universidad de Vigo.

Minka, T. P. 2002. Estimating a Gamma distribution. Microsoft Research, Cambridge, UK, Tech. Rep.

Montesinos, L., A. 2011. Estudio del AIC y BIC en la selección de modelos de vida con datos censurados. Tesina de Maestría. Centro de Investigación en Matemáticas, A.C. Guanajuato, Gto.

Moreno, R. S., Aguilar, A. R., Hernández, E. F., \& Soto, F. P. 2011. Aplicaciones de la distribución weibull en ingeniería de confiabilidad. Memoria del XXI Coloquio Mexicano de Economía Matemática y Econometría, p. 148.

Navarrete, L., C.F. 2008. Distribuição de probabilidade e dimensionamento amostral para tamanho de partícula em gramíneas forrageiras. Teses de Mestra. Universidade de São Paolo. Escola Superior de Agricultura Luiz de Queiroz. 
Ramírez, Á. S. 2000. Criterios para la selección de modelos estadísticos. Banco Central de Costa Rica. DIE-NT-07-00.

Räisänen, J. 2009. Probability distributions of monthlyto-annual mean temperature and precipitation in a changing climate (CES Climate Modelling and Scenarios Deliverable D2.4, task I). Department of
Physics, P.O. Box 48, FI-00014 University of Helsinki, Finland. AVAILABLE FROM: http:// www. atm.helsinki.fi/ jaraisan/CES_D2.4/ CES D2.4 task1.html

Schwarz. G. 1978. Estimating the dimension of a model. The Annals of Statistics, 6(2), pp. 461464. 